\title{
The onset of dissipation in the kinematic dynamo
}

\author{
Jean-Luc Thiffeault ${ }^{\mathrm{a})}$ and Allen H. Boozer \\ Department of Applied Physics and Applied Mathematics, Columbia University, New York, New York 10027
}

(Received 23 September 2002; accepted 21 October 2002)

\begin{abstract}
The kinematic regime of the magnetic dynamo neglects the backreaction of the magnetic field on the flow. For small magnetic diffusivity, in the early stage of evolution, there is an ideal phase where dissipative effects can also be neglected. The magnitude of the energy dissipation term is estimated (Ohmic heating), taking into account differential constraints on chaotic flows. The period of ideal evolution is roughly doubled over an estimate without constraints. The helicity generation terms are exponentially smaller than the energy dissipation, so that large quantities of energy are dissipated before any helicity can be created. Helicity flow is exponentially larger than net helicity generation. The constraints also lead to the existence of a singular initial condition for the magnetic field for which sizable amounts of helicity can potentially be created. (C) 2003 American Institute of Physics. [DOI: 10.1063/1.1528902]
\end{abstract}

\section{INTRODUCTION}

For many three-dimensional flows an embedded magnetic field will grow exponentially; this phenomenon is referred to as a dynamo. ${ }^{1}$ The kinematic dynamo problem consists of studying the induction equation for the field on the assumption that it does not react back on the flow (the Lorentz force is neglected). This is justified as long as the field is small enough compared to inertial forces. A good estimate of the domain of validity of the kinematic assumption is thus of paramount importance.

It was recently pointed out by Schekochihin $e t a l^{2}$ that the details of how the nonlinear Lorentz force term appears in the equations of motion are important. The Lorentz tension force only involves the gradient of the magnetic field along itself (the parallel gradient), and in smooth chaotic flows or turbulent flows at large Prandtl number that gradient is much smaller than its perpendicular counterpart. Thus, the onset of the reaction force is delayed as compared with an estimate based on the scale and magnitude of the magnetic field.

In this paper we investigate the domain of validity of the ideal evolution assumption, that the field is passively advected by the plasma with resistive effects being negligible. Because the resistivity (magnetic diffusivity) is assumed very small in dimensionless terms (i.e., the magnetic Reynolds number is very large, typically $10^{8}-10^{15}$; for example, it is of order $10^{12}$ in the solar corona), there is a sizable period of evolution where the scale of the magnetic field is such that Ohmic dissipation is unimportant. In a flow with chaotic Lagrangian trajectories the exponential stretching of fluid elements leads to growth of the amplitude of the magnetic field, so that the magnetic energy grows very rapidly during the period of ideal evolution. But in incompressible flows there is a corresponding exponential decrease via folding of the scale of magnetic field variations. These small scale variations in the field cause the Ohmic dissipation term

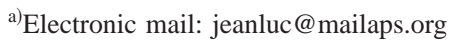

to become large, and the evolution is no longer ideal. A convenient method of estimating the importance of the nonideal (resistive) term is to compare the rate of growth of magnetic energy to that of the power dissipated by Ohmic heating. This was done in Refs. 3 and 4 where it was found that, because the power dissipation involves extra gradients of the magnetic field, the ratio of energy over power (the dissipation time scale) decreases exponentially in time at a rate $-4 \lambda$, where $\lambda$ is a typical value of the Lyapunov exponent of the flow-the mean rate of exponential stretching of fluid elements.

In the present paper we will show that these estimates are overly pessimistic. Because of the existence of differential constraints on the characteristic directions and amplitudes of stretching, the dissipation time scale decreases exponentially at a typical rate $-2 \lambda$, implying that the period of ideal evolution is twice as long as previously thought. These differential constraints originated in the work of Tang and Boozer $^{5}$ on the advection-diffusion equation, and Thiffeault and Boozer ${ }^{6}$ found a new constraint valid in three dimensions that we apply here to the kinematic dynamo. Methods from differential geometry were used to show that the derivatives along the characteristic directions of expansion of the stretching rates cannot be completely independent and have to satisfy constraints. The theory of differential constraints was recently expanded and put on a more rigorous footing by Thiffeault. ${ }^{7}$ The constraints have been tested numerically to a high degree of precision for various maps and flows. ${ }^{5-7}$

After reviewing aspects of stretching in chaotic flows in Sec. II, we calculate the energy dissipation time scale in the manner of Boozer ${ }^{3}$ in Sec. III and introduce the necessary notation along the way. The effect of differential constraints on the dissipation time scale is derived in Sec. IV. In Sec. V we investigate an intriguing consequence of the differential constraint, namely that there exists a singular initial condition for which the energy dissipation time scale does not decrease exponentially; we speculate on the physical meaning of such an initial condition. Section VI is devoted to 
deriving estimates of the amount of helicity created during the period of ideal growth, both with and without constraints and for the singular initial condition. Finally, in Sec. VII we offer some general comments on the results of the paper and discuss future work.

\section{STRETCHING IN CHAOTIC FLOWS}

In this section we review the properties of stretching in chaotic flows, as embodied by a metric tensor in Lagrangian coordinates that measures the total deformation experienced by a fluid element as it is advected and stretched by the flow.

For a given smooth velocity field $\mathbf{v}(\mathbf{x}, t)$, the trajectory of a fluid element is the solution to

$$
\dot{\mathbf{x}}(\mathbf{a}, t)=\mathbf{v}(\mathbf{x}(\mathbf{a}, t), t), \quad \mathbf{x}(\mathbf{a}, 0)=\mathbf{a},
$$

where $\mathbf{x}(\mathbf{a}, t)$ is the position at time $t$ of a fluid element that started at a at $t=0$. The initial condition labels a are called Lagrangian coordinates, and the $\mathbf{x}$ are the Eulerian coordinates. (The Eulerian coordinates are "fixed" in space and can be regarded as ordinary Cartesian coordinates.) The function $\mathbf{x}=\mathbf{x}(\mathbf{a}, t)$ is thus the transformation from Lagrangian (a) to Eulerian ( $\mathbf{x}$ ) coordinates. For a chaotic flow, this transformation gets extremely complicated as time evolves.

The Jacobian matrix of the transformation $\mathbf{x}(\mathbf{a}, t)$ is $M_{q}^{i}:=\partial x^{i} / \partial a^{q}$. The Jacobian matrix is a precise record of how an initially spherical fluid element is rotated and stretched into an ellipsoid by the flow. We are interested in describing stretching and its direction in the reference frame of a fluid element (Lagrangian frame), not the absolute rotation in Eulerian space, so we construct the metric tensor or Cauchy-Green strain tensor, ${ }^{8} g_{p q}:=\sum_{i=1}^{3} M_{p}^{i} M_{q}^{i}$, which contains only the information on the stretching of fluid elements (this can be seen, for example, by performing a singular value decomposition of $M^{7,9,10}$ ). The metric tensor is a symmetric, positive-definite matrix, so it can be diagonalized with orthogonal eigenvectors $\{\hat{\mathbf{u}}, \hat{\mathbf{m}}, \hat{\mathbf{s}}\}$, and corresponding real, positive eigenvalues $\left\{\Lambda_{u}^{2}, \Lambda_{m}^{2}, \Lambda_{s}^{2}\right\}$; we can thus write

$$
g_{p q}=\Lambda_{u}^{2} \hat{u}_{p} \hat{u}_{q}+\Lambda_{m}^{2} \hat{m}_{p} \hat{m}_{q}+\Lambda_{s}^{2} \hat{s}_{p} \hat{s}_{q} .
$$

The $\Lambda$ 's and $\{\hat{\mathbf{u}}, \hat{\mathbf{m}}, \hat{\mathbf{s}}\}$ are functions of $\mathbf{a}$ and $t$ (but see the following). The $\Lambda$ 's are called coefficients of expansion. Without loss of generality, we order them such that $\Lambda_{u}$ $>\Lambda_{m}>\Lambda_{s}$ (we assume nondegeneracy of the eigenvalues). The label " $u$ " indicates an unstable direction: after some time, we have $\Lambda_{u} \gg 1$, growing exponentially for large time. Such a direction exists if the flow has chaotic trajectories, which we assume to be the case. The label " $s$ " indicates a stable direction: after some time, we have $\Lambda_{s} \ll 1$, decreasing exponentially for long times. The intermediate direction, denoted by " $m$," can grow or decrease exponentially at a rate somewhere between that of $\Lambda_{u}$ and $\Lambda_{s}$; for steady flows (i.e., when $\mathbf{v}$ is independent of $t), \Lambda_{m}$ has only algebraic (nonexponential) behavior. ${ }^{11}$ The determinant of the metric is denoted by $g$; if $\mathbf{v}$ is incompressible $(\nabla \cdot \mathbf{v}=0)$, we have $\sqrt{g}$ $=\Lambda_{u} \Lambda_{m} \Lambda_{s}=1$.

The eigenvectors $\{\hat{\mathbf{u}}, \hat{\mathbf{m}}, \hat{\mathbf{s}}\}$ are called characteristic directions of stretching. It is well-known that these characteristic directions converge exponentially in to time-asymptotic values that depend only on the initial condition a (and the initial time for unsteady flows). ${ }^{7,12}$ We assume that these directions (on the trajectory of interest) have converged. It is also well known that the Lyapunov exponents converge far slower than the characteristic direction. We do not assume that the finite-time Lyapunov exponents $\lambda_{\mu}(\mathbf{a}, t)$, defined by

$$
\lambda_{\mu}:=\frac{1}{t} \log \Lambda_{\mu}
$$

have converged or even that their limit as $t \rightarrow \infty$ exists; we merely suppose that $t$ is sufficiently large to have $\Lambda_{u} \gg 1$ and $\Lambda_{s} \ll 1$. This difference in magnitude between the unstable and stable directions is the basis for the approximations used in this paper.

\section{ENERGY AND CURRENT ALONG TRAJECTORIES}

Equipped with the metric tensor in diagonal form (2), we can now compute the evolution of physical quantities, such as the energy and current, along fluid trajectories. This was investigated in Refs. 3 and 4, where the relative growth rates of energy, parallel current, etc., were derived. In the present section we revisit these results; in Sec. IV we will show that they must be modified to account for differential constraints.

The evolution of a magnetic field in resistive magnetohydrodynamics (MHD) is governed by the induction equation,

$$
\frac{\partial \mathbf{B}}{\partial t}=\nabla \times(\mathbf{v} \times \mathbf{B})+\frac{\eta}{\mu_{0}} \nabla^{2} \mathbf{B},
$$

where $\mathbf{B}$ is the magnetic field, $\eta$ is the resistivity, and $\mu_{0}$ is the permeability of free space. With the help of the chain rule and the metric tensor, we can transform the magnetic induction equation (4) from Eulerian coordinates, $\mathbf{x}$, to Lagrangian coordinates, a,

$$
\left.\frac{\partial}{\partial t}\right|_{\mathbf{a}} b^{r}(\mathbf{a}, t)=\sum_{p, q=1}^{3} \frac{\eta}{\mu_{0}} \frac{\partial}{\partial a^{p}}\left[g^{p q}(\mathbf{a}, t) \frac{\partial}{\partial a^{q}} b^{r}(\mathbf{a}, t)\right],
$$

where $b^{r} / \sqrt{g}:=\sum_{i}\left(M^{-1}\right)^{r}{ }_{i} B^{i}$ is the magnetic field in the Lagrangian frame, and $g^{p q}:=\left(g^{-1}\right)^{p q}$. The $\left.\right|_{\text {a }}$ subscript on $\partial / \partial t$ is a reminder that the time derivative is taken holding a constant, as opposed to the derivative in (4) which has $\mathbf{x}$ fixed.

Equation (5) is simply a diffusion equation with anisotropic diffusivity tensor $\left(\eta / \mu_{0}\right) g^{p q}$. By construction, the velocity $\mathbf{v}$ has dropped out of the equation entirely. When $\eta$ $=0$, we have the well-known Cauchy solution $\mathbf{b}=\mathbf{b}(\mathbf{a})$, independent of time, so that $\mathbf{b}$ is the magnetic field at $t=0$. This is the classic result that in ideal MHD the magnetic field is frozen into the plasma.

In the absence of diffusivity, the magnetic field is amplified in a chaotic flow, as can be seen by considering the magnetic energy $E_{B}:=\left(1 / 2 \mu_{0}\right) B^{2}=g_{p q} b^{p} b^{q} / 2 \mu_{0} g$. From Eq. (2), the dominant part of $B^{2}$ is $\Lambda_{u}^{2}(\mathbf{b} \cdot \hat{\mathbf{u}})^{2} / g$, which in a chaotic flow grows exponentially with time. 
In Lagrangian coordinates, the induced current $\mathbf{j}$ $=\nabla \times \mathbf{B} / \mu_{0}$ is $^{3}$

$$
\mu_{0} j^{r}=\sum_{p, q, q^{\prime}} \frac{\varepsilon^{r p q}}{\sqrt{g}} \frac{\partial}{\partial a^{p}}\left(g_{q q^{\prime}} \frac{b^{q^{\prime}}}{\sqrt{g}}\right),
$$

which after using Eq. (2) and projecting along the $\{\hat{\mathbf{u}}, \hat{\mathbf{m}}, \hat{\mathbf{s}}\}$ basis becomes

$$
\begin{aligned}
\mu_{0} j_{u}= & \Lambda_{u}^{2} b_{u} \hat{\mathbf{u}} \cdot \nabla_{0} \times \hat{\mathbf{u}}-\Lambda_{m}^{2} b_{m} \hat{\mathbf{s}} \cdot\left[\nabla_{0} \log \left(\Lambda_{m}^{2}\left|b_{m}\right|\right)\right. \\
& \left.-\left(\hat{\mathbf{m}} \cdot \nabla_{0}\right) \hat{\mathbf{m}}\right]+O\left(\Lambda_{s}^{2}\right), \\
\mu_{0} j_{m}= & \Lambda_{u}^{2} b_{u} \hat{\mathbf{s}} \cdot\left[\nabla_{0} \log \left(\Lambda_{u}^{2}\left|b_{u}\right|\right)-\left(\hat{\mathbf{u}} \cdot \nabla_{0}\right) \hat{\mathbf{u}}\right]+O\left(\Lambda_{m}^{2}\right), \\
\mu_{0} j_{s}= & -\Lambda_{u}^{2} b_{u} \hat{\mathbf{m}} \cdot\left[\nabla_{0} \log \left(\Lambda_{u}^{2}\left|b_{u}\right|\right)-\left(\hat{\mathbf{u}} \cdot \nabla_{0}\right) \hat{\mathbf{u}}\right]+O\left(\Lambda_{m}^{2}\right),
\end{aligned}
$$

where $j_{u}:=(\sqrt{g} \hat{\mathbf{u}} \cdot \mathbf{j}), b_{u}:=(\hat{\mathbf{u}} \cdot \mathbf{b} / \sqrt{g})$, etc. The subscript on $\nabla_{0}$ denotes differentiation with respect to the Lagrangian coordinates, a. In Refs. 3 and 4 it was concluded that the dominant term in $g j^{2}=\Lambda_{u}^{2} j_{u}^{2}+\Lambda_{m}^{2} j_{m}^{2}+\Lambda_{s}^{2} j_{s}^{2}$ arises from $\Lambda_{u}^{2} j_{u}^{2}$, so that overall the magnitude of the current grows as $\Lambda_{u}^{6} / g^{2}$. This is an extremely rapid growth: following Ref. 3 , we define the dissipation time scale,

$$
\tau_{d}:=E_{B} / \eta j^{2},
$$

the ratio of the magnetic energy to the power dissipated in the plasma through Ohmic heating, and find that $\tau_{d}$ $\sim g \Lambda_{u}^{-4}$. Of course, because the Cauchy solution for the magnetic field is only valid for ideal evolution-before the dissipation comes into play-the time scale $\tau_{d}$ must be regarded as an indicator of the accumulation of small scale gradients. When $\tau_{d}$, which is initially large because of the small resistivity, becomes of the order of typical macroscopic time scales, the ideal evolution approximation becomes invalid. That $\tau_{d}$ evolves as $g \Lambda_{u}^{-4}$ indicates that the ideal evolution must be abandoned rather quickly, and that relatively little magnetic energy has accumulated when this time is reached. We will see in Sec. IV that the situation is not as hopeless as it appears: the actual domain of validity of ideal evolution is considerably greater than the calculation of this section indicates.

\section{THE EFFECT OF DIFFERENTIAL CONSTRAINTS}

The conclusions of Sec. III regarding the decay of $\tau_{d}$ were based on the assumption that $\hat{\mathbf{u}} \cdot \nabla_{0} \times \hat{\mathbf{u}}$ in (7) was a nonexponential function. In Refs. 6 and 7 it was shown that this is not the case: in three dimensions we must have

$$
\hat{\mathbf{u}} \cdot \nabla_{0} \times \hat{\mathbf{u}} \sim \Lambda_{u}^{-2} \max \left(\sqrt{g}, \Lambda_{m}^{2}\right) \rightarrow 0,
$$

as long as the flow is chaotic and there exists a contracting direction, $\hat{\mathbf{s}}$. Equation (11) is one example of a differential constraint in chaotic flows; it is realized with exponential accuracy in time. Because it will recur often, we define the factor

$$
\mathcal{K}:=\max \left(\sqrt{g}, \Lambda_{m}^{2}\right) .
$$

For a steady incompressible flow, the growth or decay of $\mathcal{K}$ is algebraic, not exponential, so in that case we can neglect $\mathcal{K}$ compared to exponential factors to get an estimate of growth rates.

The constraint (11) and others [see the following, Eqs. (13) and (14)] were derived in Ref. 6 using the condition that the Riemannian curvature of the metric tensor $g_{p q}$ vanishes. In Ref. 7 the constraints were derived under more general assumptions, and they were shown to be present in arbitrary dimensions; their convergence rate was also obtained. The reason why (11) holds is not readily apparent; it is due to the overconstrained nature of the Lagrangian derivatives of the characteristic directions and coefficients of expansions. ${ }^{7}$ All the constraints used in this paper have been verified numerically to a high degree of precision ${ }^{5-7}$ for a variety of maps and flows.

In three dimensions, there are two more constraints in addition to (11), ${ }^{6,7}$

$$
\begin{aligned}
& \hat{\mathbf{u}} \cdot\left(\hat{\mathbf{u}} \cdot \nabla_{0}\right) \hat{\mathbf{s}}+\hat{\mathbf{s}} \cdot \nabla_{0} \log \Lambda_{u} \sim \Lambda_{s} \rightarrow 0, \\
& \hat{\mathbf{m}} \cdot\left(\hat{\mathbf{m}} \cdot \nabla_{0}\right) \hat{\mathbf{s}}+\hat{\mathbf{s}} \cdot \nabla_{0} \log \Lambda_{m} \sim \max \left(\Lambda_{s}, \Lambda_{s}^{2} / \Lambda_{m}^{2}\right) \rightarrow 0 .
\end{aligned}
$$

If we add (13) and (14), we find after some manipulation

$$
\frac{1}{\sqrt{g}} \nabla_{0} \cdot(\sqrt{g} \hat{\mathbf{s}})-\hat{\mathbf{s}} \cdot \nabla_{0} \log \Lambda_{s} \sim \max \left(\Lambda_{s}, \Lambda_{s} / \Lambda_{m}\right) \rightarrow 0 .
$$

This is a compressible $(\sqrt{g} \neq 1)$ version of the constraint used in Ref. 4, originally derived in two dimensions in Ref. 5. In two dimensions it is the only independent constraint.

In addition, if it happens that the $\hat{\mathbf{m}}$ direction (the middle eigenvalue) is a contracting direction (i.e., $\Lambda_{m}$ decreases exponentially for large times), then there is a fourth constraint,

$$
\hat{\mathbf{u}} \cdot\left(\hat{\mathbf{u}} \cdot \nabla_{0}\right) \hat{\mathbf{m}}+\hat{\mathbf{m}} \cdot \nabla_{0} \log \Lambda_{u} \sim \Lambda_{m} \rightarrow 0, \quad \text { for } \Lambda_{m} \ll 1 .
$$

Thus there are three constraints, (11), (13), and (14), if $\Lambda_{m}$ is a neutral or stretching direction, and four if $\Lambda_{m}$ is a contracting direction. For a generic three-dimensional chaotic flow satisfying the assumptions herein (that is, at least one stretching and one contracting direction), there can be no further differential constraints that involve only first derivatives of the characteristic directions and coefficients of expansion. ${ }^{7}$ It is however possible that flows with special symmetries admit additional relationships (algebraic, differential, or integrodifferential) among the characteristic directions and coefficients of expansion. One example is that for incompressible flows the coefficients of expansion obey the algebraic relationship $\Lambda_{u} \Lambda_{m} \Lambda_{s}=1$, as mentioned in Sec. II. We do not consider other relationships in the present work.

Armed with the constraint (11), we find that the growth of the component $j_{u}$, given by (7), is actually proportional to $\mathcal{K}$ defined by Eq. (12), and not $\Lambda_{u}^{2}$. This means that the dominant contribution to $j^{2}$ comes from (8),

$$
\begin{aligned}
\mu_{0}^{2} g j^{2}= & \mu_{0}^{2}\left(\Lambda_{u}^{2} j_{u}^{2}+\Lambda_{m}^{2} j_{m}^{2}+\Lambda_{s}^{2} j_{s}^{2}\right) \\
= & \Lambda_{u}^{4} \Lambda_{m}^{2}\left(b_{u} \hat{\mathbf{s}} \cdot\left[\nabla_{0} \log \left(\Lambda_{u}^{2}\left|b_{u}\right|\right)-\left(\hat{\mathbf{u}} \cdot \nabla_{0}\right) \hat{\mathbf{u}}\right]\right)^{2} \\
& +O\left(\Lambda_{u}^{2} \max \left(\Lambda_{m}^{4}, g^{2}\right)\right),
\end{aligned}
$$


so that $j^{2} \sim \Lambda_{u}^{4} \Lambda_{m}^{2} / g^{2}$ rather than the value $\Lambda_{u}^{6} / g^{2}$ derived in Sec. III. With this new scaling the revised dissipation time scale becomes $\tau_{d} \sim g \Lambda_{u}^{-2} \Lambda_{m}^{-2}$ instead of $g \Lambda_{u}^{-4}$. Considerably more magnetic energy can thus be created before resistivity comes into play. In fact, for the typical case of $\Lambda_{m}$ nonexponential (i.e., for steady flows) the time for ideal evolution is roughly doubled. Of course because the production of magnetic energy is exponential in the ideal phase, this leads to an exponentially larger amount of energy.

The modifications to the growth of the parallel current when the constraint is taken into account are even more drastic. By parallel current, we mean the part of $\mathbf{j}$ along the magnetic field,

$$
j_{\|}^{2}:=(\mathbf{j} \cdot \mathbf{B})^{2} / B^{2} .
$$

In Refs. 3 and 4 it was concluded that $\mathbf{j}$ aligns with $\mathbf{B}$ because the dominant part of $j^{2}$ comes from $j_{u}$, so that $j_{\|}^{2}$ $\sim \Lambda_{u}^{6} / g^{2}$. But if we apply the constraint (11), we find instead that $j_{\|}^{2} \sim \Lambda_{u}^{2} \mathcal{K}^{2} / g^{2}$. This is a radically different growth rate: for a steady incompressible flow (i.e., $\Lambda_{m}$ nonexponential and $g=1$ ), the ratio of the previous to the new result is $\Lambda_{u}^{6} / \Lambda_{u}^{2}=\Lambda_{u}^{4}$. The most important implication is that we now have $j_{\|}^{2} \ll j_{\perp}^{2}$, so that the current does not align with the magnetic field. In terms of length scales, we have that the scale of variation of $\mathbf{B}$ along itself, $\ell_{\|}$, is much greater than the perpendicular variation, $\ell_{\perp}$. The dominant contribution to $j_{\|}$comes from a mixture of $j_{u}$ and $j_{m}$, whereas the dominant contribution to $j^{2}$ comes from $j_{m}$.

Note that when we speak of alignment of two vectors we are expressing this in terms of their scalar product. The scalar product is invariant under coordinate transformations, including the transformation between Eulerian and Lagrangian coordinates. ${ }^{13}$ This means that the alignment occurs both in the Lagrangian and Eulerian frames. This is the power of the Lagrangian trajectory approach: the behavior of the vectors is easily derived in Lagrangian coordinates, and the results automatically apply to the Eulerian frame if expressed in terms of scalar quantities.

\section{A SINGULAR INITIAL CONDITION}

So far we have only made use of the constraint (11). In the present section we offer an application of the other constraints. If we apply the constraints (14) and (13), respectively, to the $j_{u}$ and $j_{m}$ components of the current, Eqs. (7) and (8), we find

$$
\begin{aligned}
\mu_{0} j_{u}= & \Lambda_{u}^{2} b_{u} \hat{\mathbf{u}} \cdot \nabla_{0} \times \hat{\mathbf{u}}-\Lambda_{m}^{2} b_{m} \hat{\mathbf{s}} \cdot \nabla_{0} \log \left(\tilde{\Lambda}_{m}\left|b_{m}\right|\right) \\
& +\Lambda_{u}^{-1} O\left(\Lambda_{m}, 1, \Lambda_{m}^{3} / \sqrt{g}\right), \\
\mu_{0} j_{m}= & \Lambda_{u}^{2} b_{u} \hat{\mathbf{s}} \cdot \nabla_{0} \log \left(\tilde{\Lambda}_{u}\left|b_{u}\right|\right)+\frac{\Lambda_{u}}{\Lambda_{m}^{2}} O\left(\Lambda_{m}, 1, \Lambda_{m}^{3} / \sqrt{g}\right),
\end{aligned}
$$

where we have made use of the fact that, asymptotically, the Lagrangian derivatives of $\log \Lambda_{u}, \log \Lambda_{m}$, and $\log g$ satisfy $^{7}$

$$
\hat{\mathbf{s}} \cdot \nabla_{0} \log \Lambda_{u}=\hat{\mathbf{s}} \cdot \nabla_{0} \log \tilde{\Lambda}_{u}+O\left(\Lambda_{s}, \Lambda_{s} / \Lambda_{m}, \Lambda_{m} / \Lambda_{u}\right),
$$

$$
\begin{aligned}
& \hat{\mathbf{s}} \cdot \nabla_{0} \log \Lambda_{m}=\hat{\mathbf{s}} \cdot \nabla_{0} \log \tilde{\Lambda}_{m}+O\left(\Lambda_{s}, \Lambda_{s} / \Lambda_{m}, \Lambda_{m} / \Lambda_{u}\right), \\
& \hat{\mathbf{s}} \cdot \nabla_{0} \log g=\hat{\mathbf{s}} \cdot \nabla_{0} \log \tilde{g}+O\left(\Lambda_{s}, \Lambda_{s} / \Lambda_{m}\right),
\end{aligned}
$$

with $\tilde{\Lambda}_{u}, \tilde{\Lambda}_{m}$, and $\tilde{g}$ time-independent functions of $\mathbf{a}$, unique up to a multiplicative constant. Equations (21)-(23) reflect the contraction of coordinates along the $\hat{\mathbf{s}}$ direction, which leads to exponential convergence of Lagrangian derivatives; these convergence rates were derived in detail in Ref. 7.

From (20), we see that there are two special initial conditions for $b_{u}$. The first is to simply let $b_{u} \equiv 0$, but this is not relevant here because then the magnetic energy does not grow at the rate $\Lambda_{u}$. A more interesting possibility is to let

$$
\mathbf{b} \cdot \hat{\mathbf{u}}=c \widetilde{g}^{1 / 2} \widetilde{\Lambda}_{u}^{-1},
$$

where $c$ is a constant and $\tilde{g}$ and $\widetilde{\Lambda}_{u}$ are defined in (21) and (23). The $\tilde{g}^{1 / 2}$ factor appears in (24) because of the definition $b_{u}:=(\hat{\mathbf{u}} \cdot \mathbf{b} / \sqrt{g})$. Then the first term in (20) vanishes, and we find that the leading-order behavior of the current is

$$
j^{2} \sim \Lambda_{u}^{2} \mathcal{J} / g^{2}, \quad \mathcal{J}:=\max \left(\mathcal{K}^{2}, g \Lambda_{m}^{-2}\right),
$$

in contrast to $\Lambda_{u}^{4} \Lambda_{m}^{2} / g^{2}$ for a generic initial condition. For an asymptotic rate such as (25), the dissipation time scale (10) is $\tau_{d} \sim g / \mathcal{J}$; for a steady incompressible flow (i.e., $\Lambda_{m}$ nonexponential and $g=1$ ), we have $\tau_{d} \sim 1$. Hence, for this special initial condition the dissipation time can be of order unity. We thus have an exponentially growing magnetic field whose growth can be sustained for a relatively long time before dissipation becomes important.

As for the parallel current $j_{\|}^{2}$, it remains unchanged for the singular initial condition and is equal to $\Lambda_{u}^{2} \mathcal{K}^{2} / g^{2}$; its main contribution is from $j_{u}$. Thus, asymptotically, the singular initial condition has the magnetic field and current aligned. (In terms of the scale of variation of the magnetic field, we have $\ell_{\|} \sim \ell_{\perp}$.)

The existence of a singular initial condition is made possible by the constraints (11) and (13)-(14), but it is also crucial that the Lagrangian derivatives (21) and (23) converge to well-defined time-asymptotic values, which is the case for almost all initial conditions. ${ }^{7}$ Otherwise it would not be feasible to ask for an initial condition of the form (24) since $\mathbf{b}$ is constant in time (until the diffusive regime is reached).

The function $\tilde{\Lambda}_{u}$ has the following properties: (i) it is time independent; (ii) it is smoothly varying along $\hat{\mathbf{s}}$; and (iii) it is "rough" along $\hat{\mathbf{u}}$. Point (i) was discussed in the previous paragraph and makes possible the existence of the singular initial condition. Points (ii) and (iii) are discussed in detail (Ref. 7). The roughness of $\widetilde{\Lambda}_{u}$ is directly related to the separation of trajectories in chaotic system, and can be expected to persist down to the resistive scale.

We shall comment further on the singular initial condition in Sec. VII. 


\section{HELICITY GENERATION}

In the present section we turn our attention to the production of magnetic helicity. The magnetic helicity $\mathcal{H}$ $:=\mathbf{A} \cdot \mathbf{B}$, where $\mathbf{A}$ is the magnetic potential with $\nabla \times \mathbf{A}=\mathbf{B}$, evolves according to

$$
\frac{\partial \mathcal{H}}{\partial t}+\nabla \cdot(\mathbf{v} \mathcal{H})=-\eta(2 \mathbf{j} \cdot \mathbf{B}-\nabla \cdot(\mathbf{A} \times \mathbf{j})) .
$$

(Some terms were absorbed by an appropriate choice of gauge.) Helicity can only be created or destroyed if $\eta \neq 0$. [We refer to the terms on the right-hand side of (26) as the helicity generation terms, but they are not sign-definite and can also destroy helicity.] The second helicity generation term in (26) creates no net helicity, as it vanishes upon integration over the entire volume (for suitable boundary conditions). However, it can move helicity from one point to another: it represents a flux of helicity, so that the total helicity flux is $\mathbf{v} \mathcal{H}-\eta \mathbf{A} \times \mathbf{j}$.

The magnetic energy $E_{B}=B^{2} / 2 \mu_{0}$ is dissipated by Ohmic heating in the plasma at a rate given by $\eta j^{2}$. We want to examine the relative magnitude along fluid trajectories of local helicity generation as given by (26) and energy dissipation through Ohmic heating $\eta j^{2}$. This will help determine whether the net helicity in a region is due to relative transport (helicity flux) or creation (the $2 \eta \mathbf{j} \cdot \mathbf{B}$ term). It is thought that helicity generation is an important ingredient for the creation of a large-scale magnetic field, ${ }^{14}$ in part because helicity decays on a slower time scale than energy ${ }^{15}$ so its presence ensures a long-lived magnetic field. It is also important because of its implications for the topology (knottedness) of magnetic field lines. ${ }^{16-18}$ Given the new results involving the constraints, it is reasonable to ask if there is sufficient time in the ideal energy evolution phase to create a significant amount of helicity. Put another way, when the Ohmic heating term becomes of order one, how large are the helicity generation terms?

Following the trajectory of a fluid element, (26) becomes

$$
\left.\frac{\partial}{\partial t}\right|_{\mathbf{a}}(\mathbf{A} \cdot \mathbf{b})=-\eta\left(2 \mathbf{j} \cdot \mathbf{b}-\nabla_{0} \cdot(\mathbf{A} \times \mathbf{j})\right),
$$

where all the variables are expressed in the Lagrangian frame and $\mathcal{H}=\mathbf{A} \cdot \mathbf{b} / \sqrt{g}$. If we form the ratio of the helicity generation terms on the right-hand side of (27) and power dissipation $\eta j^{2}$, the resistivity $\eta$ cancels, making these two ratios convenient measures of the relative strengths of the effects. We can now ascertain whether the helicity generation terms become important before ideal evolution ends, and find which helicity generation term is largest.

We start by comparing the first term on the right-hand side of (27) to the Ohmic heating term $\eta j^{2}$, ignoring the constraint (11):

$$
\frac{\mathbf{j} \cdot \mathbf{b}}{j^{2}}=\sqrt{g} \frac{j \| B}{j^{2}} \sim \sqrt{g} \frac{\Lambda_{u}^{3}}{g} \frac{\Lambda_{u}}{\sqrt{g}} \frac{g^{2}}{\Lambda_{u}^{6}}=g \Lambda_{u}^{-2} .
$$

The helicity generation term is typically exponentially small compared to energy dissipation. Including the constraint (11) does not improve matters:

$$
\frac{\mathbf{j} \cdot \mathbf{b}}{j^{2}} \sim \sqrt{g} \frac{\Lambda_{u} \mathcal{K}}{g} \frac{\Lambda_{u}}{\sqrt{g}} \frac{g^{2}}{\Lambda_{u}^{4} \Lambda_{m}^{2}}=g \Lambda_{u}^{-2} \Lambda_{m}^{-2} \mathcal{K},
$$

so that the helicity generation term is still typically exponentially smaller than Ohmic heating.

For the singular initial condition of Sec. V, the ratio of helicity generation to Ohmic dissipation yields

$$
\frac{\mathbf{j} \cdot \mathbf{b}}{j^{2}} \sim \sqrt{g} \frac{\Lambda_{u} \mathcal{K}}{g} \frac{\Lambda_{u}}{\sqrt{g}} \frac{g^{2}}{\Lambda_{u}^{2} \mathcal{J}}=\frac{g \mathcal{K}}{\mathcal{J}} .
$$

The ratio is nonexponential (i.e., order one) for a steady incompressible flow. This opens the possibility of creating a sizable amount of helicity before Ohmic dissipation becomes important.

The second term on the right-hand side of (27) — the flux of magnetic helicity in Lagrangian coordinates-is given by

$$
\nabla_{0} \cdot(\mathbf{A} \times \mathbf{j})=\sum_{\mu} \nabla_{0} \cdot\left[\Lambda_{\mu}^{2}\left(\mathbf{A} \times \hat{\mathbf{e}}_{\mu}\right) j_{\mu} / \sqrt{g}\right]
$$

where $\mu=\{u, m, s\}$ and $\{\hat{\mathbf{e}}\}:=\{\hat{\mathbf{u}}, \hat{\mathbf{m}}, \hat{\mathbf{s}}\}$. Because A does not acquire a special orientation with respect to $\hat{\mathbf{e}}_{\mu}$, upon expansion the divergence in (31) will generate terms of the form $\hat{\mathbf{u}} \cdot \nabla_{0} \log \Lambda$, and these grow proportionally to $\Lambda_{u} \cdot{ }^{7}$ Hence we find

$$
\nabla_{0} \cdot(\mathbf{A} \times \mathbf{j}) \sim \frac{\Lambda_{u}}{\sqrt{g}} \sum_{\mu} \Lambda_{\mu}^{2} j_{\mu} .
$$

The overall growth rate thus depends on whether the constraint (11) is used, because this affects which component of $j_{\mu}$ is largest. Without the constraint we have both $j_{u}$ and $j_{m}$ proportional to $\Lambda_{u}^{2} / \sqrt{g}$; then the dominant contribution in (32) comes from $j_{u}$, yielding

$$
\nabla_{0} \cdot(\mathbf{A} \times \mathbf{j}) \sim \frac{\Lambda_{u}}{\sqrt{g}} \Lambda_{u}^{2} j_{u} \sim \Lambda_{u}^{5} / g .
$$

But the result (33) was obtained without taking differential constraints into account and is thus incorrect. Upon applying the constraint (11), we have $j_{u} \sim \mathcal{K} / \sqrt{g}$, so that

$$
\nabla_{0} \cdot(\mathbf{A} \times \mathbf{j}) \sim \Lambda_{u}^{3} \mathcal{K} / g .
$$

Taking the ratio with the Ohmic heating $\eta j^{2}$, we find that $\nabla_{0} \cdot(\mathbf{A} \times \mathbf{j}) / j^{2}$ is proportional to $g \Lambda_{u}^{-1}$ without the constraint (11), and to $g \Lambda_{u}^{-1} \Lambda_{m}^{-2} \mathcal{K}$ with the constraint. For the case of a steady incompressible flow, this helicity source term is again exponentially smaller than the power dissipation, and moreover the ratio of the two is roughly $\Lambda_{u}^{-1}$ both with and without the constraint. The rate (34) also applies to the singular initial condition, but in that case $j^{2}$ grows more slowly [Eq. (25)], allowing more accumulation of helicity before the end of ideal evolution.

If we compare the two terms (helicity creation and helicity flux) on the right-hand side of (27) to each other, we find

$$
\frac{\nabla_{0} \cdot(\mathbf{A} \times \mathbf{j})}{\mathbf{j} \cdot \mathbf{b}} \sim \Lambda_{u}
$$


TABLE I. Asymptotic behavior of physical quantities. Three cases are considered: (i) no differential constraint; (ii) constraint given by Eq. (11) used; (iii) singular initial condition of Sec. V, which uses the constraints (11)-(14). The factor $\mathcal{K}$ is defined in (12), $\mathcal{J}$ in (25).

\begin{tabular}{lcccc}
\hline \hline & No constraint & Constraint & Sing. initial cond. & Comments \\
\hline$B^{2}$ & $\Lambda_{u}^{2} / g$ & $\Lambda_{u}^{2} / g$ & $\Lambda_{u}^{2} / g$ & Magnetic energy \\
$j^{2}$ & $\Lambda_{u}^{6} / g^{2}$ & $\Lambda_{u}^{4} \Lambda_{m}^{2} / g^{2}$ & $\Lambda_{u}^{2} \mathcal{J} / g^{2}$ & Power dissipation \\
$j_{\|}^{2}$ & $\Lambda_{u}^{6} / g^{2}$ & $\Lambda_{u}^{2} \mathcal{K}^{2} / g^{2}$ & $\Lambda_{u}^{2} \mathcal{K}^{2} / g^{2}$ & Parallel current \\
$\mathbf{j} \cdot \mathbf{b} / j^{2}$ & $g \Lambda_{u}^{-2}$ & $g \Lambda_{u}^{-2} \Lambda_{m}^{-2} \mathcal{K}$ & $g \mathcal{K} / \mathcal{J}$ & Helicity creation vs power \\
$\nabla_{0} \cdot(\mathbf{A} \times \mathbf{j}) / j^{2}$ & $g \Lambda_{u}^{-1}$ & $g \Lambda_{u}^{-1} \Lambda_{m}^{-2} \mathcal{K}$ & $g \Lambda_{u} \mathcal{K} / \mathcal{J}$ & Helicity flux vs power \\
\hline \hline
\end{tabular}

in all three cases (constrained, unconstrained, and singular initial condition). We conclude that though the proper application of the constraints changes the absolute magnitude of each helicity source term in (27), when expressed as a ratio with the current their relative magnitude is unchanged. The helicity flux (second term) always tends to dominate the helicity creation (first term) as a helicity source on the righthand side of (27).

Table I summarizes the growth rates derived in previous sections and in this section for helicity generation. For a clearer picture of relative magnitudes, Table II gives the corresponding growth rates for a steady incompressible flow. The singular initial condition is the only case that allows for the possibility of significant helicity creation or flow before the dissipative regime is reached. For the other two cases (unconstrained and constrained), for very small $\eta$ the magnetic field will have built up huge gradients by the time the evolution ceases to be ideal. Exponentially large amounts of power thus seem required to create or move helicity. Even for the singular initial condition, there is no guarantee that the helicity will be created rather than destroyed, or will be concentrated rather than dispersed, since the generation and flow terms are not definite in sign. In fact, it is known that substantial cancellation of the helicity occurs, ${ }^{19}$ a fact that is not captured in our analysis.

We emphasize that the results for the unconstrained case are presented merely for comparison purposes: the constrained results always apply because differential constraints are an unavoidable geometrical consequence of the chaotic nature of the flow. ${ }^{7}$ The singular initial condition, however, only applies to situations that are initialized in the special manner given by (24). Otherwise the constrained result applies.

TABLE II. As for Table I but assuming a steady incompressible flow $\left(\Lambda_{m}\right.$ $\sim 1, g=1$ ).

\begin{tabular}{lccc}
\hline \hline & No constraint & Constraint & Sing. initial cond. \\
\hline$B^{2}$ & $\Lambda_{u}^{2}$ & $\Lambda_{u}^{2}$ & $\Lambda_{u}^{2}$ \\
$j^{2}$ & $\Lambda_{u}^{6}$ & $\Lambda_{u}^{4}$ & $\Lambda_{u}^{2}$ \\
$j_{\|}^{2}$ & $\Lambda_{u}^{6}$ & $\Lambda_{u}^{2}$ & $\Lambda_{u}^{2}$ \\
$\mathbf{j} \cdot \mathbf{b} / j^{2}$ & $\Lambda_{u}^{-2}$ & $\Lambda_{u}^{-2}$ & 1 \\
$\nabla_{0} \cdot(\mathbf{A} \times \mathbf{j}) / j^{2}$ & $\Lambda_{u}^{-1}$ & $\Lambda_{u}^{-1}$ & $\Lambda_{u}$ \\
\hline \hline
\end{tabular}

\section{DISCUSSION}

Using Lagrangian coordinates, we have revised earlier estimates of the magnitude of energy dissipation (Sec. IV) and helicity generation (Sec. VI). The estimates were corrected for differential constraints on the rates and directions of stretching. The inclusion of constraints leads roughly to a doubling of the period of ideal evolution, for the case of a steady incompressible flow. We also found after using constraints that the current does not align with the magnetic field in the ideal phase, in contrast with the results of Refs. 3 and 4 where it was concluded that it did. The constraints thus have a deep implication on the evolution of the magnetic field, at least in the ideal phase. We did not discuss estimates of the magnitude of the Lorentz force on the flow, as this was done in Ref. 4 and the constraints do not appear to modify those results.

In spite of the constraints, we find that the helicity generation terms are exponentially smaller than the energy dissipation, so that creation of significant amounts of helicity cannot occur in the period of ideal energy growth (when resistivity can be neglected). Our analysis does not take into account the fact that the helicity created will be fractal in nature, and so very little of it will contribute to a large-scale magnetic field, as pointed out by Gilbert. ${ }^{19}$ This suggests a close inspection of the energy dissipation in numerical simulations, since current dynamo models could require vast amounts of energy to create helicity. The implications of helicity transport are the subject of several recent investigations. ${ }^{20-22}$

We found (Sec. V) that there exists a special initial condition with desirable properties: (i) the power dissipated is of the same order as the magnetic energy; (ii) the helicity generation terms are comparable to the power dissipated. This opens the possibility of a flow creating a considerable amount of energy and helicity before reaching the end of the ideal evolution regime. The physical relevance of the singular initial condition is uncertain. We cannot expect a realistic system to have an initial magnetic field corresponding that particular condition. The part of the initial condition that is not singular will reach the dissipation scale much sooner, so the ideal evolution hypothesis becomes invalid. But does the singular part of the initial condition survive, or is it dissipated away? An analysis involving the full, dissipative equation is necessary to establish this and has not yet been performed. If it does survive, it could be closely related to the 
"strange eigenfunction" (i.e., a fractal) that occurs in the freely decaying advection-diffusion problem ${ }^{23}$ and its analog for the dynamo. ${ }^{1}$

\section{ACKNOWLEDGMENTS}

We thank the referee for constructive comments.

This work was supported by the National Science Foundation and the Department of Energy under a Partnership in Basic Plasma Science grant, No. DE-FG02-97ER54441.

${ }^{1}$ S. Childress and A. D. Gilbert, Stretch, Twist, Fold: The Fast Dynamo (Springer, Berlin, 1995).

${ }^{2}$ A. Schekochihin, S. Cowley, J. Maron, and L. Malyshkin, Phys. Rev. E 65, 016305 (2002).

${ }^{3}$ A. H. Boozer, Astrophys. J. 394, 357 (1992).

${ }^{4}$ X. Z. Tang and A. H. Boozer, Phys. Plasmas 7, 1113 (2000).

${ }^{5}$ X. Z. Tang and A. H. Boozer, Physica D 95, 283 (1996).

${ }^{6}$ J.-L. Thiffeault and A. H. Boozer, Chaos 11, 16 (2001).

${ }^{7}$ J.-L. Thiffeault, Physica D 172, 139 (2002).
${ }^{8}$ J. M. Ottino, The Kinematics of Mixing: Stretching, Chaos, and Transport (Cambridge University Press, Cambridge, 1989).

${ }^{9}$ J. M. Greene and J. S. Kim, Physica D 24, 213 (1987).

${ }^{10}$ K. Geist, U. Parlitz, and W. Lauterborn, Prog. Theor. Phys. 83, 875 (1990).

${ }^{11}$ J.-P. Eckmann and D. Ruelle, Rev. Mod. Phys. 57, 617 (1985).

${ }^{12}$ I. Goldhirsch, P. Sulem, and S. A. Orszag, Physica D 27, 311 (1987).

${ }^{13}$ In Lagrangian coordinates, by scalar product of two vectors $\mathbf{v}$ and $\mathbf{w}$ we mean $\Sigma_{p, q} g_{p q} v^{p} w^{q}$. The presence of the metric $g$ ensures the invariance of the scalar. In Eulerian coordinates we mean $\Sigma_{i, j} \delta_{i j} v^{i} w^{j}$, where $\delta$ is the Euclidean matrix. In the text, $\mathbf{v} \cdot \mathbf{w}$ always means $\Sigma_{i} v^{i} w^{i}$, which is not a scalar in Lagrangian coordinates.

${ }^{14}$ A. H. Boozer, Phys. Fluids B 5, 2271 (1993).

${ }^{15}$ J. B. Taylor, Phys. Rev. Lett. 33, 1139 (1974).

${ }^{16}$ L. Woltjer, Proc. Natl. Acad. Sci. U.S.A. 44, 489 (1958).

${ }^{17}$ H. K. Moffatt, J. Fluid Mech. 35, 117 (1969).

${ }^{18}$ H. K. Moffatt and M. R. E. Proctor, J. Fluid Mech. 154, 493 (1985).

${ }^{19}$ A. D. Gilbert, Geophys. Astrophys. Fluid Dyn. 96, 135 (2002).

${ }^{20}$ E. G. Blackman and G. B. Field, Astrophys. J. 534, 984 (2000).

${ }^{21}$ E. T. Vishniac and J. Cho, Astrophys. J. 550, 752 (2001).

${ }^{22}$ A. Bradenburg, Astrophys. J. 550, 824 (2001).

${ }^{23}$ R. T. Pierrehumbert, Chaos, Solitons Fractals 4, 1091 (1994). 